Article

\title{
Biological Aggressiveness of Subclinical No-Mass Ductal Carcinoma In Situ (DCIS) Can Be Reflected by the Expression Profiles of Epithelial- Mesenchymal Transition Triggers
}

\author{
Bartlomiej Szynglarewicz ${ }^{1,2, *(1)}$, Piotr Kasprzak ${ }^{3}$, Piotr Donizy ${ }^{4}$, Przemyslaw Biecek ${ }^{5}$, \\ Agnieszka Halon ${ }^{4}$ and Rafal Matkowski ${ }^{1,2}$ \\ 1 Breast Unit, Department of Surgical Oncology, Lower Silesia Oncology Center, 53-413 Wroclaw, Poland; \\ rafal.matkowski@umed.wroc.pl \\ 2 Department of Oncology, Faculty of Postgraduate Medical Training, Wroclaw Medical University, \\ 53-413 Wroclaw, Poland \\ 3 Department of Breast Imaging, Lower Silesia Oncology Center, 53-413 Wroclaw, Poland; kasp@poczta.onet.pl \\ 4 Department of Pathomorphology and Oncological Cytology, Wroclaw Medical University, 53-413 Wroclaw, \\ Poland; chir.onk.2@dco.com.pl (P.D.); ahalon2@gmail.com (A.H.) \\ 5 Faculty of Mathematics and Information Science, Warsaw University of Technology, 00-662 Warsaw, Poland; \\ przemyslaw.biecek@gmail.com \\ * Correspondence: szynglarewicz.b@dco.com.pl; Tel.: +48-713-689-391
}

Received: 14 November 2018; Accepted: 6 December 2018; Published: 7 December 2018

\begin{abstract}
Epithelial-mesenchymal transitions (EMTs) have been recently implicated in the process of cancer progression. The aim of this study was to assess how the preoperative expression patterns of EMT biomarkers correlate with the risk of postoperative invasion in ductal carcinoma in situ (DCIS) found on stereotactic breast biopsies. N-cadherin, Snail1, and secreted protein acidic and rich in cysteine (SPARC) immunoreactivity was observed in $8 \%, 62 \%$, and $38 \%$ of tumors, respectively. Snail1 and SPARC expressions were significantly related to $\mathrm{N}$-cadherin expression and to each other. The postoperative upgrading rate was associated with a positive preoperative expression of all biomarkers. Significance of Snail1 and SPARC persisted in multivariate analysis, but the impact of SPARC on invasion was more significant. When these two EMT triggers were considered together, the risk of invasion did not significantly differ between the subtypes of DCIS with single positive expression (SPARC - /Snail1+ vs. SPARC+/Snail1-). However, it was significantly lower in single-positive DCIS when compared to lesions of a double-positive profile (SPARC+/Snail1+). Moreover, there were no cases in the double-negative DCIS (SPARC-/Snail1-), with foci of infiltrating cancer found postoperatively in residual postbiopsy lesions. In contrast, DCIS with a combined high SPARC and Snaill expression (intermediate or strong) had an invasive component in $66-100 \%$ of tumors.
\end{abstract}

Keywords: ductal carcinoma in situ; epithelial-mesenchymal transition; N-cadherin; SPARC; Snail1

\section{Introduction}

Since the implementation of mammographic screening programs, the incidence of early breast cancer, in situ lesions, and borderline proliferations has increased dramatically [1]. Among them, ductal carcinoma in situ (DCIS), which is usually diagnosed during a stereotactic biopsy of suspicious microcalcifications detected on a mammography, accounts for approximately $20 \%$ of all breast cancers [2,3]. Moreover, studies of the natural course of DCIS managed with biopsies only (no curative 
therapy) suggest that up to $75 \%$ of patients do not develop an invasive disease during follow-up after 15-25 years [4-6]. Thus, optimal management and clinical decision-making in newly diagnosed DCIS cases is nowadays a considerable challenge, and the need for a biology-based treatment strategy is postulated [1]. Identification of molecular and biological markers can help to tailor a more individual approach, avoid overtreatment in indolent DCIS, and improve outcomes in aggressive subtypes.

Epithelial-mesenchymal transition (EMT) is a dynamic process whereby an immotile epithelial cell temporarily changes its morphology to a spindle-like shape and acquires the migratory and invasive properties of a mesenchymal cell. This allows for invasion through the basal membrane as well as the extracellular matrix and, ultimately, migration to distant sites, where it reverts back into the epithelial phenotype [7]. EMT is implicated in a number of different biological processes, including embryonic development, tissues repair, and wound healing. EMT has also been shown to play a crucial role in cancer progression, invasion, and metastasis [8]. Molecular changes during EMT are regulated by several transcription factors called EMT-inducers (Snail1, Snail2, ZEB1, ZEB2, TWIST, FOXC2, E47, TCF4), regulators of the extracellular matrix that facilitate the degradation of the basal membrane and surrounding tissues (SPARC, TGF- $\beta$, EGF, FGF), as well as molecules from related pathways (e.g., MAPK, P13K, Wnt, NF-kB, Notch, Hedgehog) [9]. Epithelial cells are immobile due to the well-constructed cytoskeleton and the strong cell-cell and cell-extracellular matrix adhesions, which consist of adherens junctions, tight junctions, and desmosomes [9]. To initiate a cell phenotype interconversion, key components of intercellular junctions such as E-cadherin, claudins, occludins, and desmosomes are downregulated by EMT inducers. Loss of E-cadherin expression and the concomitant upregulation or de novo expression of N-cadherin, referred to as a cadherin switch, is considered a hallmark of EMT [10].

In our previous papers, we reported a significant association between the presence of final invasion following surgery in DCIS, diagnosed with a stereotactic vacuum-assisted biopsy as a pure in situ lesion, and a preoperative expression of molecules that trigger the early steps of EMT: Transcription factor Snail1, a direct E-cadherin repressor, and the multifunctional de-adhesive protein SPARC (secreted protein acidic and rich in cysteine), a key modulator of the tumor microenvironment.

The aim of the present study was to investigate whether the predictive value of individual SPARC and Snail1 expression could be improved by the additional assessment of $\mathrm{N}$-cadherin as well as by the complex analysis of the combined expression profiles of the aforementioned EMT biomarkers.

\section{Results}

\subsection{Clinical Features}

Baseline characteristics are presented in Table 1. Among the clinical and pathological variables, there was a significant correlation between the type and distribution of microcalcifications as well as between the nuclear grade and comedonecrosis, as we reported previously [11,12]. Casting-type microcalcifications most commonly presented a regional distribution pattern. Conversely, powdery and crushed stone-like microcalcifications exhibited a clustered and grouped distribution pattern, respectively $(p<0.0001)$. Traditional histological analysis revealed a direct relationship between the presence of comedonecrosis and the severity of the lesion's grade. A stepwise decrease in the incidence of comedonecrosis could be observed from high (91\%) through intermediate (56\%) to low (45\%) grade lesions $(p<0.0001)$. 
Table 1. Baseline characteristics.

\begin{tabular}{|c|c|}
\hline Variable & Results \\
\hline \multicolumn{2}{|c|}{ Patient age } \\
\hline Median/range & $59 / 41-88$ \\
\hline Mean \pm SD & $58.4 \pm 7.9$ \\
\hline \multicolumn{2}{|c|}{ Microcalcification type, $n(\%)$} \\
\hline Powdery & $70(34)$ \\
\hline $\begin{array}{l}\text { Crushed } \\
\text { stone-like }\end{array}$ & $107(51)$ \\
\hline Casting-type & $32(15)$ \\
\hline \multicolumn{2}{|c|}{ Microcalcification distribution, $n(\%$} \\
\hline Cluster & $145(69)$ \\
\hline Group & $44(21)$ \\
\hline Regional & $20(10)$ \\
\hline \multicolumn{2}{|c|}{ Nuclear grade, $n(\%)$} \\
\hline Low & $110(52)$ \\
\hline Intermediate & $66(32)$ \\
\hline High & $33(16)$ \\
\hline \multicolumn{2}{|c|}{ Comedonecrosis, $n(\%)$} \\
\hline Absent & $93(45)$ \\
\hline Present & $116(55)$ \\
\hline \multicolumn{2}{|c|}{ N-cadherin expression, $n(\%)$} \\
\hline Negative & $192(92)$ \\
\hline Positive & $17(8)$ \\
\hline \multicolumn{2}{|c|}{ Snaill expression, $n(\%)$} \\
\hline Negative & $80(38)$ \\
\hline Positive & $129(62)$ \\
\hline Weak & $57(27)$ \\
\hline Intermediate & $43(21)$ \\
\hline Strong & $29(14)$ \\
\hline \multicolumn{2}{|c|}{ SPARC expression, $n(\%)$} \\
\hline Negative & $130(62)$ \\
\hline Positive & $79(38)$ \\
\hline Weak & $33(16)$ \\
\hline Intermediate & $20(10)$ \\
\hline Strong & $26(12)$ \\
\hline
\end{tabular}

\subsection{EMT Biomarkers in Preoperative Pathology (Biopsy Specimens)}

In this study, $8 \%$ (17) of DCIS was positive for $\mathrm{N}$-cadherin. Snail1 expression was found in $62 \%$ of lesions (129). Positive immunoreactivity was weak, intermediate, and strong in $27 \%$ (57), $21 \%$ (43), and $14 \%$ (29) of cases, respectively. SPARC upregulation was observed in $38 \%$ of tumors (79). Positive staining was weak, intermediate, and strong in 16\% (33), 10\% (20), and $12 \%$ (26) of patients, respectively. $\mathrm{N}$-cadherin expression was significantly related to the distribution of microcalcifications (positivity most common in clustered $<1 \mathrm{~cm}$, least in regional $>2 \mathrm{~cm} ; p=0.033$ ). As we presented before, the correlation between SPARC and the type of microcalcifications was very close to the significance limit (positivity most common in casting type, least common in powdery; $p=0.061$ ) [11]. Expression of Snail1 was also significantly associated with the morphology of microcalcifications (similarly to SPARC, most common in casting type, least common in powdery; $p=0.041$ ). However, it was also significantly related to the presence of comedonecrosis (72\% of Snail1-positive tumors in comedo-DCIS, $48 \%$ in noncomedo; $p=0.001$ ) [12]. No other significant correlation between the investigated biomarkers and 
the clinical and pathological variables was observed. Among EMT factors, upregulation of N-cadherin was significantly associated with Snail1 and SPARC expression ( $p=0.019$ and $p=0.017$, respectively). Positive Snail1 and positive SPARC were related to each other with a very high significance $(p<0.0001)$. The correlation is presented in Table 2.

Table 2. Significant correlation between N-cadherin, Snail1, and secreted protein acidic and rich in cysteine (SPARC) expression; $n(\%)$.

\begin{tabular}{|c|c|c|c|c|c|c|}
\hline & \multicolumn{2}{|c|}{ N-Cadherin } & \multicolumn{2}{|c|}{ SPARC } & \multicolumn{2}{|c|}{ Snail1 } \\
\hline & Negative & Positive & Negative & Positive & Negative & Positive \\
\hline \multicolumn{7}{|c|}{ N-cadherin } \\
\hline Negative & - & - & $124(65)$ & $68(35)$ & $78(41)$ & $114(59)$ \\
\hline Positive & - & - & $6(35)$ & $1(65)$ & $2(12)$ & $15(88)$ \\
\hline \multicolumn{7}{|c|}{ SPARC } \\
\hline Negative & $124(95)$ & $6(5)$ & - & - & $66(51)$ & $64(49)$ \\
\hline Positive & $68(86)$ & $11(14)$ & - & - & $14(18)$ & $65(82)$ \\
\hline \multicolumn{7}{|c|}{ Snail1 } \\
\hline Negative & $78(97)$ & $2(3)$ & $66(82)$ & $14(18)$ & - & - \\
\hline Positive & $114(88)$ & $15(12)$ & $64(49)$ & $65(51)$ & - & - \\
\hline
\end{tabular}

\subsection{Final Invasion in Postoperative Pathology (Surgical Specimens)}

Invasion was present on final pathology in 34 patients, which gave a postoperative upgrading rate of $16 \%$. Patient age did not significantly influence that risk. Neither mammographic appearance of microcalcifications (distribution, morphology) nor traditional pathologic features (nuclear grade, comedonecrosis) had significant predictive value. Upgrading to invasive cancer was more common in N-cadherin-positive DCIS ( $35.3 \%$ vs. $14.6 \%$ ). The difference was very close to the significance limit $(p=0.0608)$. The risk of final invasion was significantly higher in Snail1-positive DCIS compared to Snail1-negative DCIS: $25 \%$ versus $3 \%$, respectively $(p<0.0001)$. Among Snail1-positive tumors, there was a stepwise increase in the upgrading rate from poor immunoreactivity $(9 \%)$ through intermediate $(26 \%)$ to strong staining $(55 \%)$. The correlation was of very high significance $(p<0.0001)$ [12]. The risk of final invasion was also significantly enhanced in SPARC-positive DCIS when compared to SPARC-negative DCIS: $38 \%$ versus $3 \%$, respectively $(p<0.0001)$. Likewise, for Snail1, a stepwise risk increase from poor immunoreactivity (3\%) through intermediate $(50 \%)$ to strong staining $(73 \%)$ was observed. That association was also very significant $(p<0.0001)$ [11].

Multivariate analysis demonstrated that both Snail1 and SPARC were significant and independent predictive factors for postoperative invasion $(p<0.001$ and $p<0.0001$, respectively). However, the impact of SPARC upregulation on invasion was more significant than Snaill expression: Odds Ratio $3.940,95 \%$ Confidence Interval 2.502-6.637, versus 2.143, and 1.248-3.822, respectively. The association between preoperative features of DCIS and the presence of invasive disease on final pathology is presented in Table 3.

When these two EMT triggers were considered together, the risk of invasion did not significantly differ between the subtypes of DCIS with single positive expression of either SPARC or Snail1 (SPARC-/Snail1+ vs. SPARC+/Snail1-: $6.25 \%$ vs. $14.29 \%, p=0.306$ ). However, the risk of invasion was significantly lower in single-positive DCIS when compared to lesions of a double-positive profile (SPARC+/Snail1+: $7.7 \%$ vs. $43.1 \%, p<0.0001$ ). This significant difference persisted when the subtypes of single-positive DCIS were compared to double-positive DCIS separately: SPARC - /Snail1+ versus SPARC+/Snail1 + $(6.25 \%$ vs. $43.08 \%, p<0.0001)$ and SPARC+/Snail1 - versus SPARC+/Snail1 + $(14.3 \%$ vs. $43.08 \%, p=0.044)$, respectively. Moreover, DCIS with a combined high SPARC and Snail1 expression (intermediate/strong) had an invasive component in $66-100 \%$ of tumors. In contrast, there was no case 
(0/66) among the double-negative DCIS (SPARC - /Snail1-), with foci of infiltrating cancer found postoperatively in the residual postbiopsy lesion. Invasion rates are presented in Table 4.

Table 3. Association between preoperative features and final invasion on surgical specimens.

\begin{tabular}{|c|c|c|c|c|}
\hline \multirow{2}{*}{ Variable } & \multicolumn{2}{|c|}{ Postoperative Invasion } & \multirow{2}{*}{$\begin{array}{l}\text { Univariate } \\
\text { Analysis } p\end{array}$} & \multirow{2}{*}{$\begin{array}{c}\text { Multivariate } \\
\text { Analysis } p\end{array}$} \\
\hline & Absent $n(\%)$ & Present $n(\%)$ & & \\
\hline \multicolumn{5}{|c|}{ Microcalcifications type } \\
\hline Powdery & $59(84)$ & $11(16)$ & \multirow{3}{*}{0.645} & \multirow{3}{*}{-} \\
\hline $\begin{array}{l}\text { Crushed } \\
\text { stone-like }\end{array}$ & $91(85)$ & $16(15)$ & & \\
\hline Casting-type & $25(78)$ & $7(22)$ & & \\
\hline \multicolumn{5}{|c|}{ Microcalcifications distribution } \\
\hline Cluster & $123(85)$ & $22(15)$ & \multirow{3}{*}{0.771} & \multirow{3}{*}{-} \\
\hline Group & $36(76)$ & $8(24)$ & & \\
\hline Regional & $16(80)$ & $4(20)$ & & \\
\hline \multicolumn{5}{|c|}{ Nuclear grade } \\
\hline Low & $95(86)$ & $15(14)$ & \multirow{3}{*}{0.417} & \multirow{3}{*}{-} \\
\hline Intermediate & $52(79)$ & $14(21)$ & & \\
\hline High & $28(85)$ & $5(15)$ & & \\
\hline \multicolumn{5}{|c|}{ Comedonecrosis } \\
\hline Absent & $80(86)$ & $13(14)$ & \multirow[b]{2}{*}{0.520} & \multirow{2}{*}{-} \\
\hline Present & $95(82)$ & $21(18)$ & & \\
\hline \multicolumn{5}{|c|}{ N-cadherin expression } \\
\hline Negative & $162(84)$ & $30(16)$ & \multirow{2}{*}{0.061} & \multirow{2}{*}{-} \\
\hline Positive & $13(76)$ & $4(24)$ & & \\
\hline \multicolumn{5}{|c|}{ Snail1 expression } \\
\hline Negative & $78(97)$ & $2(3)$ & \multirow{5}{*}{$<0.0001$} & \multirow{5}{*}{0.007} \\
\hline Positive & $97(75)$ & $32(25)$ & & \\
\hline Weak & $52(91)$ & $5(9)$ & & \\
\hline Intermediate & $32(74)$ & $11(26)$ & & \\
\hline Strong & $13(45)$ & $16(55)$ & & \\
\hline \multicolumn{5}{|c|}{ SPARC expression } \\
\hline Negative & $126(97)$ & $4(3)$ & \multirow{5}{*}{$<0.0001$} & \multirow{5}{*}{$<0.0001$} \\
\hline Positive & $49(62)$ & $30(38)$ & & \\
\hline Weak & $32(97)$ & $1(3)$ & & \\
\hline Intermediate & $10(50)$ & $10(50)$ & & \\
\hline Strong & $7(27)$ & $19(73)$ & & \\
\hline \multicolumn{5}{|c|}{ Patient age } \\
\hline Median (range) & $59(41-88)$ & $58.5(43-75)$ & 0.980 & - \\
\hline
\end{tabular}

Table 4. Invasion rate with regard to SPARC and Snail1 immunoreactivity.

\begin{tabular}{ccccc}
\hline SPARC & \multicolumn{4}{c}{ Snail1 Expression $\boldsymbol{n} \mathbf{( \% )}$} \\
\cline { 2 - 5 } Expression $\boldsymbol{n}(\mathbf{\%})$ & Negative & Weak & Intermediate & Strong \\
\hline Negative & $0(0 / 66)$ & $3 \%(1 / 38)$ & $10 \%(2 / 21)$ & $20 \%(1 / 5)$ \\
Weak & $0(0 / 9)$ & $0(0 / 9)$ & $0(0 / 9)$ & $17 \%(1 / 6)$ \\
Intermediate & $0(0 / 3)$ & $33 \%(3 / 9)$ & $75 \%(3 / 4)$ & $100 \%(4 / 4)$ \\
Strong & $100 \%(2 / 2)$ & $100 \%(1 / 1)$ & $66 \%(6 / 9)$ & $71 \%(10 / 14)$ \\
\hline
\end{tabular}




\section{Discussion}

A cadherin switch facilitates the release of cancer cells from the primary tumor, resulting in increased migratory and invasive behavior [13]. We observed that the expression of N-cadherin was more common in DCIS postoperatively upgraded to invasive cancer compared to lesions without final invasion. ElMoneim and Zaghloul found upregulation of $\mathrm{N}$-cadherin in $52 \%$ of invasive ductal cancers. It was significantly related to larger tumor size, positive nodal status, higher clinical disease stage, a poor Nottingham prognostic index, and negative estrogen receptor (ER) and progesterone receptor (PR) status. Pure invasive carcinomas were slightly more commonly, but without significance, $\mathrm{N}$-cadherin-positive than those with an intraductal component (48\%) [14]. Kovacs et al. observed $\mathrm{N}$-cadherin expression in $30 \%$ of infiltrating cancers and $40 \%$ of DCIS. In invasive tumors, there was no significant relation between $\mathrm{N}$-cadherin positivity and histological grade, lesion size, lymph node status, ER/PR status, and epidermal growth factor receptor (EGFR) expression. However, among positive DCIS, there were no low-grade lesions (75\% high grade, $25 \%$ intermediate). Unfortunately, due to the small number of in situ lesions, no significant conclusion could be drawn [15]. In a large study of Aleskandarany and coworkers, a cadherin switch was more frequently detected in triple negative and human epidermal growth factor receptor (HER)2-positive subtypes of invasive nonluminal breast cancer than in the luminal subtype. There were also significant differences regarding prognosis and patient survival [16]. Nagi and colleagues reported N-cadherin expression in $76 \%$ of micropapillary cancers with high propensity for lymphatic infiltration, whereas the other types of cancer demonstrated an expression of only $52 \%$. Staining scores were higher in invasive tumors compared to DCIS, and this effect was more dramatic in micropapillary carcinomas. The authors concluded that $\mathrm{N}$-cadherin contributed to breast cancer progression and was associated with tumor aggressiveness and metastatic potential [17]. Choi et al. demonstrated a significantly higher expression of $\mathrm{N}$-cadherin in invasive cancers than in pure DCIS (8.7\% vs. $3.3 \%)$. Similarly, the loss of E-cadherin and upregulation of the other EMT biomarkers, such as $\beta$-catenin and smooth muscle actin, was observed [18]. In the meta-analysis of Luo et al., $\mathrm{N}$-cadherin expression was found to be a useful predictor of unfavorable overall survival [19].

In the present study, the relation between $\mathrm{N}$-cadherin and postoperative invasion was not significant, but close to the limit. The lack of statistical significance could have been caused by an insufficient proportion of $\mathrm{N}$-cadherin positive tumors required to obtain robust statistics. The small number of $\mathrm{N}$-cadherin-positive lesions may have been responsible for the relatively low rate of postoperative upgrading in the current study $(16 \%)$ when compared to other series, including our previous report [20]. This could also have been influenced by the fact that we studied cases with typical clinical and radiological features (nonpalpability, microcalcifications without mass, absence of other findings on imaging (e.g., architectural distortion, asymmetric density)) of early DCIS limited to intraductal proliferation and rarely associated with the presence of an infiltrating component.

The risk of final invasion was significantly and independently related to both EMT triggers, Snail1 and SPARC. Snail1 displays a broad spectrum of biological functions: Regulation of cell movement and adhesion, cell proliferation and survival, immune suppression, and generation of stem cell properties [21]. Snail1 triggers EMT by direct repression of the transcription of the E-cadherin gene [22]. Since it is an E-cadherin repressor inducing a cadherin switch, its overexpression is a step before the acquisition of $\mathrm{N}$-cadherin. Therefore, it is not surprising that during the very early phase of ductal cancer progression, as was the case in our study, expression of Snail1 better determined the risk of invasion than $\mathrm{N}$-cadherin. Although Snail1 is normally absent in healthy mammary epithelial cells, it often becomes activated during breast cancer progression and is observed in about $80 \%$ of microdissected human invasive ductal cancers [23,24]. The analysis of tumor development using the MMTV-PyMT (Mouse Mammary Tumor Virus - Polyomavirus Middle T-antigen) transgenic model of mammary tumor formation, which mirrors the multistep progression of human breast cancer, demonstrated that Snail1 expression was related to potent EMT activation and acquisition of aggressive features [24]. Snail1 is closely associated with a tumor-initiating cell phenotype that is responsible 
for metastatic dissemination and clinical relapse in a variety of cancers [24-26]. In MMTV-PyMT carcinoma-derived pB1.3G cells and MDA-MB-231 (M.D. Anderson Metastatic Breast cancer cell line established from a pleural effusion of a 51-year-old female with highly aggressive and poorly differentiated triple-negative ductal cancer) human breast cancer cells, the knockdown of Snail1 attenuates primary tumor growth, strongly suppresses its metastatic spreading, and induces the acquisition of epithelial traits in a reverse epithelial-mesenchymal transition process [24]. Moreover, Snail1 knockdown significantly suppresses tumor initiation in most human breast cancer cell lines [24].

There are several challenges in reconstructing and analyzing an EMT regulatory network [27]. EMT, at the tissue level, can be recognized by the detection of the downregulation of specific epithelial biomarkers and the upregulation of specific mesenchymal biomarkers. However, the acquisition of a full mesenchymal state is rarely detected in vivo. This is due to various cells being at different stages of EMT at any given time $[27,28]$. The expression of EMT markers is temporally and spatially coordinated through the activation of an entire network of transcription factors that initiates and orchestrates the EMT process [27-29]. Morphological changes during EMT reflect the actuation of a specific molecular program, activated by juxtacrine and paracrine signals derived from the tumor microenvironment, that induces hierarchical, multilayer signaling networks [27,30]. EMT regulation by the microenvironment is extremely complex, resulting in a heterogeneous activation of several cellular pathways [27]. Numerous regulators involved in the activation of EMT have been identified. SPARC, as a key modulator of the local environment, is one such mediator. It regulates cellular differentiation, proliferation, and migration; influences cell polarity, shape, and attachment; has an impact on the activity of growth factors and membrane permeability; and is involved in stromal remodeling by regulating the expression of extracellular matrix metalloproteinases as well as collagen fibrillogenesis and deposition [31,32]. In the specific tumor microenvironment, SPARC may facilitate the degradation of the basement membrane barrier and contribute to the conversion from in situ to infiltrating cancer [24]. Our findings support the notion that gaining invasive properties is a complex process with a multidirectional cross-interaction of tumor cells and stroma.

In multivariate analysis, the presence of a postoperative infiltrating component was better predicted by preoperative SPARC than Snail1: However, both factors were significant. Separate investigations resulted in invasion risk stratification from 3\% (negative) through $38 \%$ (positive) to $73 \%$ (strong expression) for SPARC, and from 3\% (negative) through 25\% (positive) to $55 \%$ (strong expression) for Snail1 [11,12]. Complex analysis of their expression more accurately reflected the process of gaining invasive capacity during the very early steps of cancer progression. SPARC/Snail1 expression profiles defined the subtypes of DCIS with significantly different risks of final invasion. It allowed for the identification of completely indolent (no risk of invasive component) and extremely aggressive (66-100\% risk of invasive component) subtypes of DCIS. A clinical implication of this is the possibility of a more tailored approach and selective surgery. Sentinel node biopsy during breast surgery can be considered in high-risk patients. In contrast, it can be avoided in low-risk women. Moreover, in selected cases, indications for breast resection can be redefined (i.e., in double-negative patients without a visible residual postbiopsy lesion, a close radiological follow-up may be a valuable option).

With regard to the invasion-metastasis cascade, the transition from an in situ to an infiltrating phenotype is an essential step in tumor metastasis. However, the question of whether the acquisition of invasive propensity by DCIS can really predict the course of disease remains open. On the one hand, significant correlations between the expression of SPARC and unfavorable clinical outcomes and a worse prognosis in DCIS has been observed [33]. Significant associations between Snail1 expression and poor overall and disease-free survival has also been reported [34]. On the other hand, a sequential model of breast cancer progression is sometimes challenged. Narod and Sopik questioned the paradigm that breast cancer passes through several stages and claimed that invasion is not a necessary step for metastasis. They proposed an alternate theory, a parallel model wherein there is a small pool of cancer stem cells that have metastatic potential and synchronously disseminate through 
several routes: To the breast tissue, to the lymph nodes, and to distant organs [35]. According to this theory, cancer cells disseminating to the breast rise and make up the bulk of the tumor mass, but they are not a source of distant metastases. However, since there is still no equivocal evidence supporting either of the progression theories, some emphasize that there are probably still other unknown cancer transition pathways and that numerous mechanisms may coexist [36].

The lack of analysis of E-cadherin was a weakness of our study. N-cadherin expression was found in relatively few cases and a concomitant investigation of E-cadherin could better reflect a cadherin switch. However, since Snail1 is a direct repressor of E-cadherin, its expression and E-cadherin downregulation are closely related to each other. Therefore, the likelihood that the analysis of E-cadherin expression, in addition to Snail1, could have improved the extremely significant predictive value of Snail1 expression in our series is disputable. Snail1 is a transcription factor, and its expression is expected to be predominantly localized in the nucleus. Surprisingly, we observed exclusively cytoplasmic staining. This is not easy to explain and has been thoroughly discussed elsewhere [12]. Briefly, nuclear expression is considered better than cytoplasmic expression or Snail1 mRNA at predicting Snail1 activity, because this transcription factor is the subject of post-translational modifications that influence its stability and localization [37-39]. However, in some investigations, both cytoplasmic and nuclear expression have been found [14,39]. In other studies, similarly to us, predominantly or even exclusively cytoplasmic Snail1 reactivity was observed in preinvasive and invasive breast and colorectal cancer [40-42]. Some claim that since Snail1 displays a broad spectrum of biological functions, a subcellular localization in the cytoplasm may not be definitively associated with a total loss of its function [42]. In addition, there are probably several more Snail1-regulated processes waiting to be discovered [21].

Our study also had some other important limitations. First, this was just an observational study, and no comparison with other EMT biomarkers was performed. Second, assessment of immunohistochemical staining is always more or less related to the limited intra- and inter-observer reproducibility. Third, some findings were based on the comparison of small groups. Even if the results were significant, their statistical power was low, and therefore it was difficult to draw any conclusive statements. Fourth, it was a single-institution case series. Taking everything into account, one cannot be sure that our results will be repeatable in a different setting.

To the best of our knowledge, this recent report is the first study addressing the predictive value of the combined expression of EMT biomarkers during the very early steps of breast cancer progression (i.e., a transition from in situ to invasive disease) performed on specimens from a preoperative stereotactic biopsy. We successfully identified the different subtypes of DCIS with varying infiltrating potential, from an indolent proliferation to an extremely aggressive lesion, which allows for more individual postbiopsy decision making and a better-designed therapy.

\section{Materials and Methods}

\subsection{Patients}

We studied 209 consecutive patients with pure DCIS diagnosed via percutaneous stereotactic vacuum-assisted breast biopsy due to suspicious microcalcifications in the years 2004-2014. All of them had breast imaging reporting and data system (BIRADS) category 4 or 5 microcalcifications without a mass or architectural distortion, lack of invasion, or microinvasion ( $\leq 1 \mathrm{~mm}$ in the longest diameter) on a postbiopsy pathological examination, and also an absence of any other breast malignancy or borderline lesion. Biopsies were completed under digital mammography guidance using a designated prone table unit (Mammotest Plus/S, Fisher Imaging, Denver, CO, USA) with a 10-G needle (EnCore Breast Biopsy System, SenoRx Inc., Irvine, CA, USA or EnCore Enspire Breast Biopsy System, C.R. Bard Inc., Tempe, AZ, USA). All procedures were performed by a single breast-dedicated radiologist (PK) at the same breast care unit and according to the same standardized protocol in order to assure quality control. The technical details of the biopsy as well as a description of the clinical factors, mammographic 
presentation, and histological features have already been presented [11]. In each case informed consent was obtained. The study was conducted according to the Declaration of Helsinki principles and approved by the Independent Ethics Committee of the Wroclaw Medical University (UMED KB-376; 19 Oct 2010) and Institutional Review Board (NDOK/668; 6 August 2012).

\subsection{Immunohistochemistry}

All slides of formalin-fixed and paraffin-embedded tissue specimens obtained from biopsies were hematoxylin-eosin-stained and then examined by two board-certified pathologists experienced in breast cancer (A.H., P.D.). All immunohistochemical evaluations were performed without knowledge of tumor and patient characteristics. In all cases, pure DCIS without an invasive or microinvasive component was reported. Expression of EMT biomarkers was assessed in luminal epithelial cells of DCIS. In each case, immunoreactivity was scored by the two study pathologists, without conflicting observations. Immunohistochemical determination of $\mathrm{N}$-cadherin expression was completed using a mouse monoclonal antibody (NC-17; catalog code: NBP1-41353; dilution: 1:100, Novus Biologicals LCC, Littleton, CO 80120, USA). It was performed on 4- $\mu$ m-thick paraffin sections mounted on silanized slides (code number S 3003, DAKO, Glostrup, Denmark), which were then subjected to deparaffinization, rehydration, and heat-induced epitope unmasking performed using PT Link, with incubation in EnVision ${ }^{\mathrm{TM}}$. Target Retrieval Solution for $20-40 \mathrm{~min}$ at $97^{\circ} \mathrm{C}$. Autostainer Link was used to perform an immunological test using the detection reagent DakoEnVision ${ }^{\mathrm{TM}}$ FLEX/HRP (SM802). Expression was assessed as negative in cases of no staining (Figure 1) or only staining of up to $20 \%$ of cells, and positive when more than $20 \%$ of the analyzed cells were stained (Figure 2 ). Due to the small number of positive cases, we did not stratify the reactivity into weak, intermediate, and strong staining.

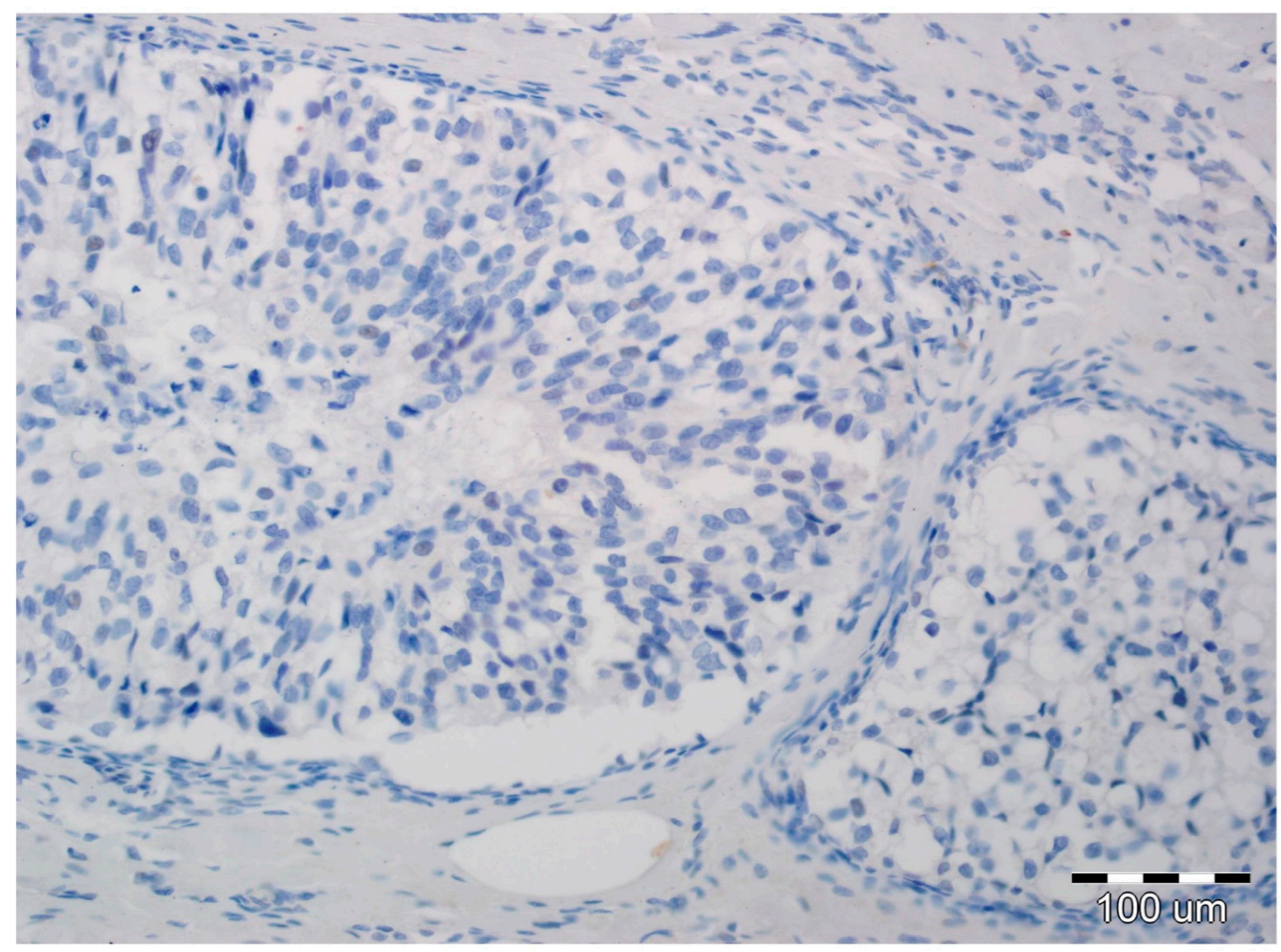

Figure 1. Ductal carcinoma in situ with lack of $\mathrm{N}$-cadherin expression in luminal epithelial cells and the stromal compartment (hematoxylin, $\times 200$ ). 


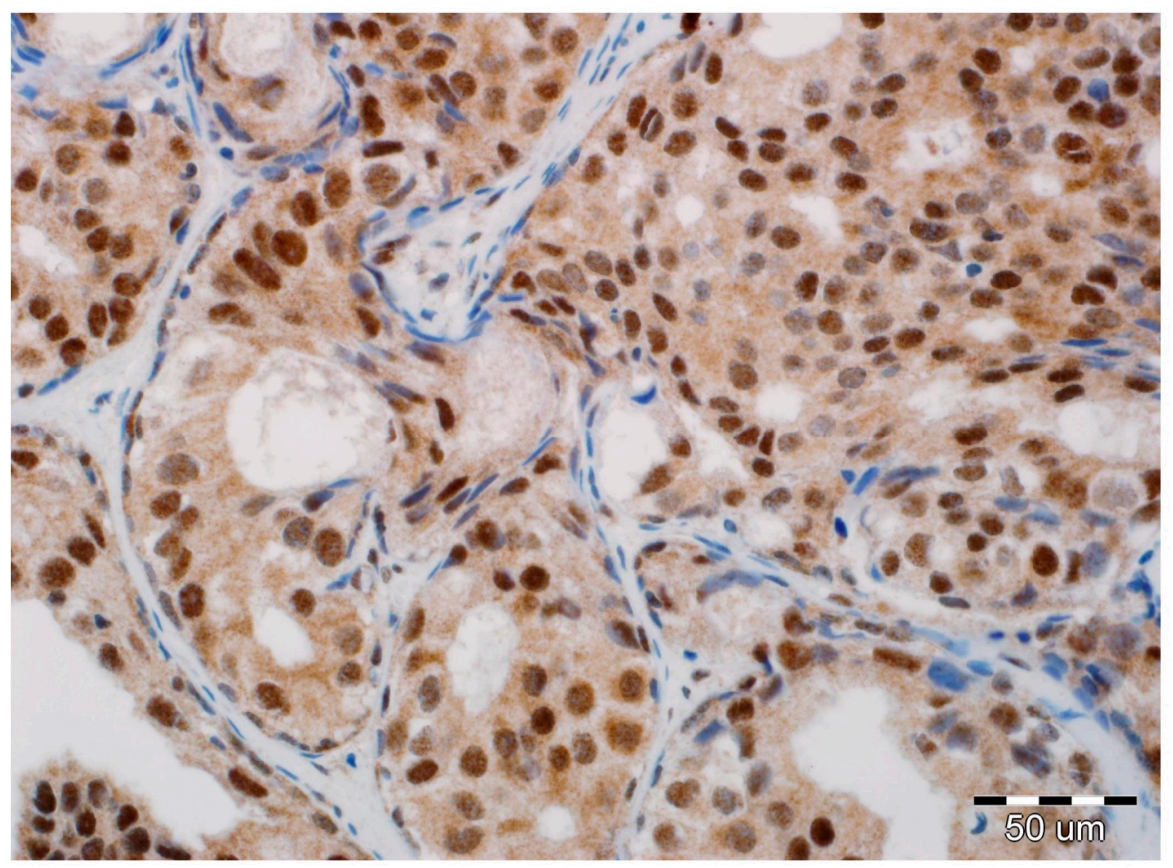

Figure 2. Positive expression of N-cadherin in luminal epithelial cells of ductal carcinoma in situ (DCIS). No reaction in stromal fibroblasts and myoepithelial cells (hematoxylin, $\times 400$ ).

Details of the immunohistochemistry of SPARC and Snail1 have been presented elsewhere [11,12]. For both, a semiquantitative method was used to evaluate the expression as negative ( 0 , no staining), weak ( 1 , either diffuse weak staining or strong staining in $<10 \%$ of analyzed cells), intermediate ( 2 , either diffuse intermediate staining or strong staining in $\geq 10 \%$ to $30 \%$ of analyzed cells), and strong (3, defined as strong staining of $>30 \%$ of analyzed cells). Strong expression of Snail1 and SPARC in DCIS luminal epithelial cells is presented in Figures 3 and 4, respectively.

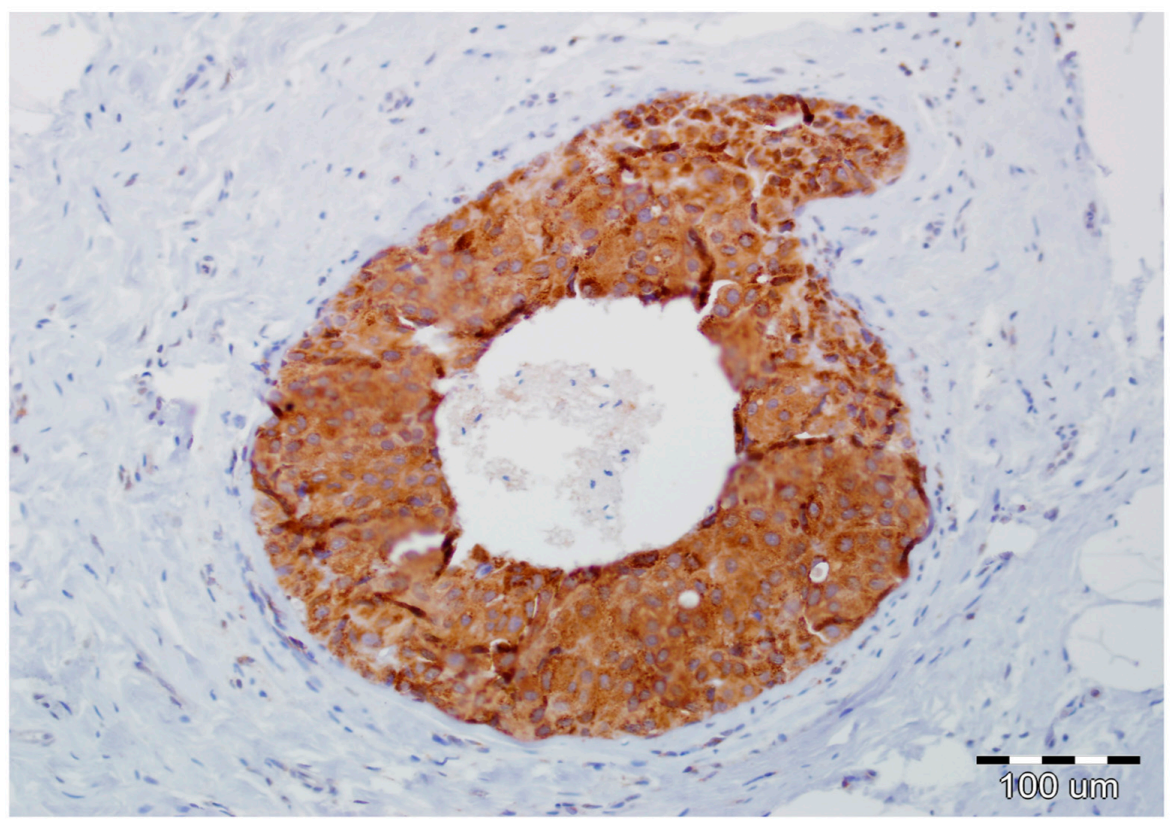

Figure 3. Strong expression of Snail1 in DCIS luminal epithelial cells and lack of its reactivity in myoepithelial cells and stromal fibroblasts (hematoxylin, $\times 200$ ). 


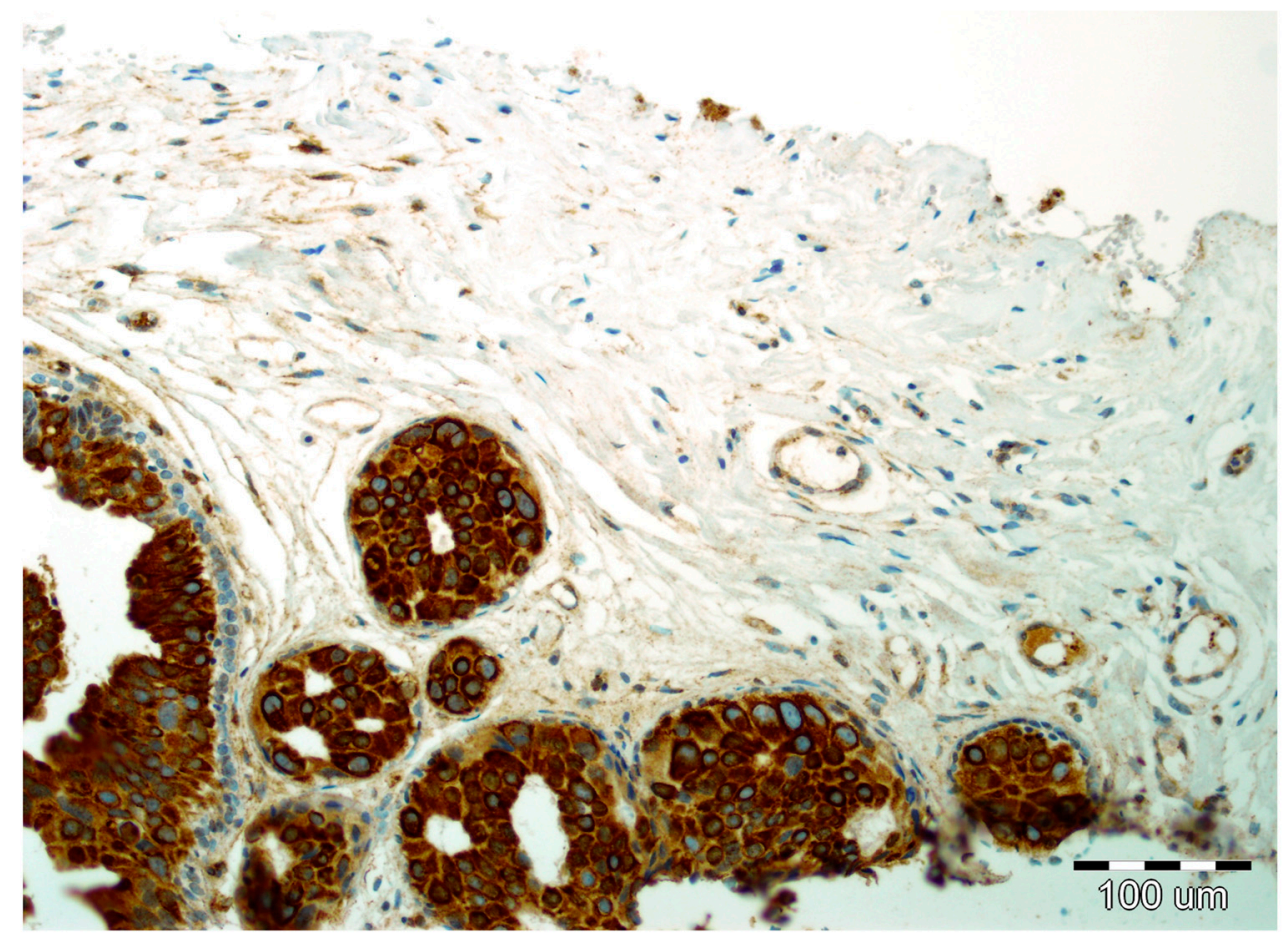

Figure 4. Strong expression of SPARC in DCIS luminal epithelial cells and focal reactivity in the stromal compartment with lack of reactivity in myoepithelial cells (hematoxylin, $\times 200$ ).

\subsection{Statistical Analysis}

Data was collected prospectively and entered into a computer database. Upgrading to invasive disease on final pathology of the postoperative specimen was calculated. A Kruskal-Wallis one-way analysis of variance was used to verify the relation between the expression of EMT biomarkers and categorical variables, whereas a Spearman's correlation coefficient was used for the continuous variables. The association between postoperative invasion and preoperative features was assessed using a chi-square test for categorical variables and a nonparametric Wilcoxon test for continuous variables. Multivariate analysis was performed with a multiple logistic regression. A $p$-value of 0.05 was considered significant. Statistical analysis was performed by a professional statistician (P.B.) with R-software ver. 3.2 (free environment for statistical computing and graphics).

\section{Conclusions}

The biology of DCIS is still not well understood. It is a heterogenic disease, and a significant proportion of these lesions will never lead to invasive breast cancer [43]. Therefore, risk stratification is essential to make better-informed clinical decisions $[43,44]$. We demonstrated that SPARC and Snail1 were better predictors of postoperative invasion than $\mathrm{N}$-cadherin. Moreover, using their combined expression profiles, indolent and very aggressive subtypes of DCIS could be preoperatively identified, which can help to tailor optimal management. Since DCIS represents an increasing proportion of newly diagnosed neoplastic breast lesions, it is vital to prevent the overtreatment of patients in cases of harmless DCIS, but concurrently to provide optimal therapy for potentially hazardous DCIS [44].

Author Contributions: B.S. conceived the study, interpreted the data, carried out the literature research, and prepared the manuscript. P.K. completed image-guided biopsies and collected the data. P.D. and A.H. reviewed pathological specimens and performed immunohistochemical analysis. B.S. and R.M. were responsible for surgical treatment. P.B. performed statistical analysis. R.M. was involved in the study design, coordinated data interpretation, and supervised the composition of the paper. All authors critically revised the manuscript and approved the final version.

Funding: This study was financed through a statutory subsidy by the Minister of Science and Higher Education as a part of the research grant ST.C280.17.010 (record number in the Simple System). 
Acknowledgments: The authors would like to thank Ewa Kowalska for data collection and management. We also thank Jacob Bronowicki for his kind assistance in revising the English of this manuscript.

Conflicts of Interest: The authors declare no conflicts of interest.

$\begin{array}{ll}\text { Abbreviations } \\ \text { DCIS } & \text { Ductal carcinoma in situ } \\ \text { EMT } & \text { Epithelial-mesenchymal transition } \\ \text { SPARC } & \text { Secreted protein acidic and rich in cysteine } \\ \text { BIRADS } & \text { Breast imaging reporting and data system } \\ \text { ER } & \text { Estrogen receptor } \\ \text { PR } & \text { Progesterone receptor } \\ \text { EGFR } & \text { Epidermal growth factor receptor } \\ \text { HER2 } & \text { Human epidermal growth factor receptor } 2\end{array}$

\section{References}

1. Sagara, Y.; Julia, W.; Golshan, M.; Toi, M. Paradigm Shift toward Reducing Overtreatment of Ductal Carcinoma in Situ of Breast. Front. Oncol. 2017, 7, 192. [CrossRef] [PubMed]

2. Virnig, B.A.; Shamliyan, T.; Tuttle, T.M.; Kane, R.L.; Wilt, T.J. Diagnosis and management of ductal carcinoma in situ (DCIS). Evid. Rep. Technol. Assess. 2009, 185, 1-549.

3. Virnig, B.A.; Tuttle, T.M.; Shamliyan, T.; Kane, R.L. Ductal carcinoma in situ of the breast: A systematic review of incidence, treatment, and outcomes. J. Natl. Cancer Instit. 2010, 102, 170-178. [CrossRef] [PubMed]

4. Page, D.L.; Dupont, W.D.; Rogers, L.W.; Landenberger, M. Intraductal carcinoma of the breast: Follow-up after biopsy only. Cancer 1982, 49, 751-758. [CrossRef]

5. Page, D.L.; Dupont, W.D.; Rogers, L.W.; Jensen, R.A.; Schuyler, P.A. Continued local recurrence of carcinoma 15-25 years after a diagnosis of low grade ductal carcinoma in situ of the breast treated only by biopsy. Cancer 1995, 76, 1197-1200. [CrossRef]

6. Sanders, M.E.; Schuyler, P.A.; Dupont, W.D.; Page, D.L. The natural history of low-grade ductal carcinoma in situ of the breast in women treated by biopsy only revealed over 30 years of long-term follow-up. Cancer 2005, 103, 2481-2484. [CrossRef] [PubMed]

7. Kim, D.H.; Xing, T.; Yang, Z.; Dudek, R.; Lu, Q.; Chen, Y.H. Epithelial Mesenchymal Transition in Embryonic Development, Tissue Repair and Cancer: A Comprehensive Overview. J. Clin. Med. 2017, 7, 1. [CrossRef]

8. Brabletz, T.; Kalluri, R.; Nieto, M.A.; Weinberg, R.A. EMT in cancer. Nat. Rev. Cancer 2018, 18, $128-134$. [CrossRef]

9. Jie, X.X.; Zhang, X.Y.; Xu, C.J. Epithelial-to-mesenchymal transition, circulating tumor cells and cancer metastasis: Mechanisms and clinical applications. Oncotarget 2017, 8, 81558-81571. [CrossRef]

10. Ashaie, M.A.; Chowdhury, E.H. Cadherins: The superfamily critically involved in breast cancer. Curr. Pharm. Des. 2016, 22, 616-638. [CrossRef] [PubMed]

11. Szynglarewicz, B.; Kasprzak, P.; Donizy, P.; Biecek, P.; Halon, A.; Matkowski, R. Ductal carcinoma in situ on stereotactic biopsy of suspicious breast microcalcifications: Expression of SPARC (Secreted Protein, Acidic and Rich in Cysteine) can predict postoperative invasion. J. Surg. Oncol. 2016, 114, 548-556. [CrossRef]

12. Szynglarewicz, B.; Kasprzak, P.; Donizy, P.; Biecek, P.; Halon, A.; Matkowski, R. Epithelial-mesenchymal transition inducer Snail1 and invasive potential of intraductal breast cancer. J. Surg. Oncol. 2017, 116, 696-705. [CrossRef] [PubMed]

13. Kourtidis, A.; Lu, R.; Pence, L.J.; Anastasiadis, P.Z. A central role for E-cadherin signaling in cancer. Exp. Cell. Res. 2017, 358, 78-85. [CrossRef]

14. ElMoneim, H.M.A.; Zaghloul, N.M. Expression of e-cadherin, n-cadherin and snail and their correlation with clinicopathological variants: an immunohistochemical study of 132 invasive ductal breast carcinomas in Egypt. Clinics 2011, 66, 1765-1771. [PubMed]

15. Kovacs, A.; Dhillon, J.; Walker, R.A. Expression of P-cadherin, but not E-cadherin or N-cadherin, relates to pathological and functional differentiation of breast carcinomas. Mol. Pathol. 2003, 56, 318-322. [CrossRef] 
16. Aleskandarany, M.A.; Negm, O.H.; Green, A.R.; Ahmed, M.A.; Nolan, C.C.; Tighe, P.J.; Ellis, I.O.; Rakha, E.A. Epithelial mesenchymal transition in early invasive breast cancer: An immunohistochemical and reverse phase protein array study. Breast Cancer Res. Treat. 2014, 145, 339-348. [CrossRef] [PubMed]

17. Nagi, C.; Guttman, M.; Jaffer, S.; Qiao, R.; Keren, R.; Triana, A.; Li, M.; Godbold, J.; Bleiweiss, I.J.; Hazan, R.B. $\mathrm{N}$-cadherin expression in breast cancer: correlation with an aggressive histologic variant-Invasive micropapillary carcinoma. Breast Cancer Res. Treat. 2005, 94, 225-235. [CrossRef] [PubMed]

18. Choi, Y.; Lee, H.J.; Jang, M.H.; Gwak, J.M.; Lee, K.S.; Kim, E.J.; Kim, H.J.; Lee, H.E.; Park, S.Y. Epithelial-mesenchymal transition increases during the progression of in situ to invasive basal-like breast cancer. Hum. Pathol. 2013, 44, 2581-2589. [CrossRef] [PubMed]

19. Luo, Y.; Yu, T.; Zhang, Q.; Fu, Q.; Hu, Y.; Xiang, M.; Peng, H.; Zheng, T.; Lu, L.; Shi, H. Upregulated N-cadherin expression is associated with poor prognosis in epithelial-derived solid tumours: A meta-analysis. Eur. J. Clin. Investig. 2018, 48, e12903. [CrossRef]

20. Szynglarewicz, B.; Kasprzak, P.; Halon, A.; Matkowski, R. Preoperatively diagnosed ductal cancers in situ of the breast presenting as even small masses are of high risk for the invasive cancer foci in postoperative specimen. World J. Surg. Oncol. 2015, 13, 218. [CrossRef]

21. Wu, Y.; Zhou, B.P. Snail. More than EMT. Cell Adh. Migr. 2010, 4, 199-203. [CrossRef] [PubMed]

22. Nieto, M.A. The snail superfamily of zinc-finger transcription factors. Nat. Rev. Mol. Cell Biol. 2002, 3, 155-166. [CrossRef] [PubMed]

23. Natrajan, R.; Weigelt, B.; Mackay, A.; Geyer, F.C.; Grigoriadis, A.; Tan, D.S.; Jones, C.; Lord, C.J.; Vatcheva, R.; Rodriguez-Pinilla, S.M.; et al. An integrative genomic and transcriptomic analysis reveals molecular pathways and networks regulated by copy number aberrations in basal-like, HER2 and luminal cancers. Breast Cancer Res Treat. 2010, 121, 575-589. [CrossRef] [PubMed]

24. Ye, X.; Tam, W.L.; Shibue, T.; Kaygusuz, Y.; Reinhardt, F.; Eaton, E.; Weinberg, R.A. Distinct EMT programs control mammary stem cells and tumour-initiating cells. Nature 2015, 525, 256-260. [CrossRef] [PubMed]

25. Al-Hajj, M.; Clarke, M.H. Self-renewal and solid tumor stem cells. Oncogene 2004, 23, 7274-7282. [CrossRef] [PubMed]

26. O’Brien, C.A.; Kreso, A.; Dick, J.E. Cancer stem cells in solid tumors: an overview. Semin. Radiat. Oncol. 2009, 19,71-77. [CrossRef]

27. Stefania, D.; Vergara, D. The many-faced program of epithelial-mesenchymal transition: A system biology-based view. Front. Oncol. 2017, 7, 274. [CrossRef] [PubMed]

28. Nieto, M.A.; Huang, R.Y.; Jackson, R.A.; Thiery, J.P. EMT: 2016. Cell 2016, 166, 21-45. [CrossRef] [PubMed]

29. De Craene, B.; Berx, G. Regulatory networks defining EMT during cancer initiation and progression. Nat. Rev. Cancer 2013, 13, 97-110. [CrossRef] [PubMed]

30. Gonzalez, D.M.; Medici, D. Signaling mechanisms of the epithelial-mesenchymal transition. Sci. Signal. 2014, 7, re8. [CrossRef] [PubMed]

31. Clark, C.J.; Sage, E.H. A prototypic matricellular protein in the tumor microenvironment-Where there's SPARC, there's fire. J. Cell Biochem. 2008, 104, 721-732. [CrossRef]

32. Nagaraju, G.P.; Dontula, R.; El-Rayes, B.F.; Lakka, S.S. Molecular mechanisms underlying the divergent roles of SPARC in human carcinogenesis. Carcinogenesis 2014, 35, 967-973. [CrossRef]

33. Witkiewicz, A.K.; Freydin, B.; Chervoneva, I.; Potoczek, M.; Rizzo, W.; Rui, H.; Brody, J.R.; Schwartz, G.F.; Lisanti, M.P. Stromal CD10 and SPARC expression in ductal carcinoma in situ (DCIS) patients predicts disease recurrence. Cancer Biol. Ther. 2010, 10, 391-396. [CrossRef] [PubMed]

34. Zhang, P.; Hu, P.; Shen, H.; Yu, J.; Liu, Q.; Du, J. Prognostic role of Twist and Snail in various carcinomas: a systematic review and meta-analysis. Eur. J. Clin. Investig. 2014, 44, 1072-1094. [CrossRef] [PubMed]

35. Narod, S.A.; Sopik, V. Is invasion a necessary step for metastases in breast cancer? Breast Cancer Res. Treat. 2018, 169, 9-23. [CrossRef] [PubMed]

36. Cunha, P.O.; Ornstein, M.; Jones, J.L. Progression of Ductal Carcinoma in Situ form the Pathological Perspective. Breast Care 2010, 5, 233-239. [CrossRef] [PubMed]

37. Geradts, J.; de Herreros, A.G.; Su, Z.; Burchette, J.; Broadwater, G.; Bachelder, R.E. Nuclear Snail1 and nuclear ZEB1 protein expression in invasive and intraductal human breast carcinomas. Hum. Pathol. 2011, 42, 1125-1131. [CrossRef] [PubMed] 
38. Muenst, S.; Daster, S.; Obermann, E.C.; Droeser, R.A.; Weber, W.P.; von Holzen, U.; Gao, F.; Viehl, C.; Oertli, D.; Soysal, S.D. Nuclear expression of Snail is an independent negative prognostic factor in human breast cancer. Dis. Markers 2013, 35, 337-344. [CrossRef] [PubMed]

39. Lundgren, K.; Nordenskjold, B.; Landberg, G. Hypoxia, Snail and incomplete epithelial-mesenchymal transition in breast cancer. Br. J. Cancer 2009, 101, 1769-1781. [CrossRef]

40. Logullo, A.F.; Nonogaki, S.; Pasini, F.S.; Osorio, C.A.; Soares, F.A.; Brentani, M.M. Concomitant expression of epithelial-mesenchymal transition biomarkers in breast ductal carcinoma: association with progression. Oncol. Rep. 2010, 23, 313-320. [PubMed]

41. Gwin, K.; Contreras, A.; Tretiakova, M.; Montag, A. Expression of the epithelial mesenchymal transition regulator Snail in metaplastic breast carcinomas with chondroid differentiation. In Proceedings of the United States \& Canadian Academy of Pathology 98th Annual Meeting, Breast Pathology Session, Boston, MA, USA, 3-7 March 2009.

42. Bezdekova, M.; Brychtova, S.; Sedlakova, E.; Langova, K.; Brychta, T.; Belej, K. Analysis of Snail-1, E-cadherin and claudin-1 expression in colorectal adenomas and carcinomas. Int. J. Mol. Sci. 2012, 13, 1632-1643. [CrossRef] [PubMed]

43. Gorringe, K.L.; Fox, S.B. Ductal Carcinoma in Situ Biology, Biomarkers, and Diagnosis. Front. Oncol. 2017, 7, 248. [CrossRef] [PubMed]

44. Groen, E.J.; Elshof, L.E.; Visser, L.L.; Rutgers, E.J.T.; Winter-Warnars, H.A.O.; Lips, E.H.; Wesseling, J. Finding the balance between over- and under-treatment of ductal carcinoma in situ (DCIS). Breast 2017, 31, 274-283. [CrossRef] [PubMed]

(C) 2018 by the authors. Licensee MDPI, Basel, Switzerland. This article is an open access article distributed under the terms and conditions of the Creative Commons Attribution (CC BY) license (http://creativecommons.org/licenses/by/4.0/). 